\title{
Epicardial Ablation of Persistent Junctional Reciprocating Tachycardia in an Infant with Tachycardia Induced Cardiomyopathy
}

\author{
Keore McKenzie ${ }^{1}$ and Sunita Ferns ${ }^{1}$ \\ ${ }^{1}$ University of Florida Health Science Center Jacksonville
}

November 9, 2020

\begin{abstract}
We report a three month old infant who developed a persistent junctional reciprocating tachycardia induced cardiomyopathy that was successfully treated with radiofrequency ablation. To our knowledge this is the youngest reported patient with a successful epicardial lesion placed in a diverticulum off of the coronary sinus. We use this case to highlight how low power lesions in the coronary sinus in the youngest of patients can achieve results safely. Delineation of coronary anatomy is crucial prior to lesion deliverance.
\end{abstract}

Epicardial Ablation of Persistent Junctional Reciprocating Tachycardia in an Infant with Tachycardia Induced Cardiomyopathy

Keore Mckenzie APRN, Sunita J Ferns MD, MRCPCH (UK)

Affiliations: Department of Pediatrics, University of Florida School of Medicine, Jacksonville, FL.

Corresponding author:

Sunita J Ferns MD, MRCPCH (UK)

Associate Professor of Medicine, University of Florida School of Medicine, Jacksonville, FL, 841 Prudential Drive, Jacksonville FL 32217, USA.

Email: Sunita.ferns@jax.ufl.edu

\section{Conflict of Interest: none}

Funding : None

Word count: 1536

50 Character running head: Epicardial PJRT ablation in an infant with cardiomyopathy

Keywords : PJRT, epicardial ablation, tachycardia induced cardiomyopathy, incessant SVT, infant ablation

\section{Abstract}

We report a three month old infant who developed a persistent junctional reciprocating tachycardia induced cardiomyopathy that was successfully treated with radiofrequency ablation. To our knowledge this is the youngest reported patient with a successful epicardial lesion placed in a diverticulum off of the coronary sinus. We use this case to highlight how low power lesions in the coronary sinus in the youngest of patients can achieve results safely. Delineation of coronary anatomy is crucial prior to lesion deliverance. 


\section{Introduction}

Persistent junctional reciprocating tachycardia (PJRT) is a rare form of refractory supraventricular tachycardia (SVT) occurring in infants and children and accounts for about $1 \%$ of all SVTs in this group. ${ }^{1}$ Because of its incessant nature, if left untreated, it may lead to tachycardia induced cardiomyopathy. The circuit is PJRT is an orthodromic AV reentry with a concealed slow conducting accessory pathway as the retrograde limb of the circuit. ${ }^{2-5}$ As both limbs in a PJRT circuit are decremental, these tachycardias tend to typically occur at lower heart rates than other SVTs and can be undetected until patients present with heart failure. ECGs during SVT typically show negative $\mathrm{P}$ waves in the inferior leads with a long RP interval. ${ }^{2,3}$

Here, we report on the case of a 3 month old infant who developed a tachycardia induced cardiomyopathy and was successfully treated with radiofrequency ablation (RFA). To our knowledge this is the youngest reported patient with a successful epicardial lesion placed in the diverticulum off the coronary sinus (CS).

\section{Case Report}

A 3 month old, $5.5 \mathrm{~kg}$, male infant with incessant SVT since fetal life presented to the Emergency Room (ER) with grunting, respiratory distress and decreased feeding for two days. He had a low grade fever, runny nose and a cough two weeks prior to presentation. There were other ill contacts in the household. On examination he had signs and symptoms of heart failure with a heart rate (HR) of 150 beats per minute(bpm), respiratory rate 50 per minute and liver $3 \mathrm{~cm}$ below the costal margin. An electrocardiogram showed a long RP tachycardia and Echocardiogram revealed an ejection fraction of $22 \%$. Laboratory work was significant for a Brain Natriuretic Peptide (BNP) of 10,000 and a viral respiratory panel positive for Parainfluenza and Coronavirus NL63 but negative for all the other Coronavirus including COVID-19.

The infant was well known to the electrophysiology service for incessant SVT since fetal life. SVT was first diagnosed at 32 weeks of gestation on a fetal ultrasound with heart rates in the $180 \mathrm{~s}-200 \mathrm{~s}$. Treatment during fetal life had been attempted with flecainide and digoxin and the baby was delivered early at 35 weeks for mild fetal hydrops. In the postnatal period SVT rates were in the 200s and rate but not rhythm control (HR:140s-150s/bpm) was achieved with high doses of amiodarone $(20 \mathrm{mg} / \mathrm{kg} /$ day $)$ and procainamide $(60 \mathrm{mic} / \mathrm{kg} / \mathrm{min})$ infusions and oral sotalol. The infant was transitioned to oral flecainide, amiodarone and sotalol was switched to a beta blockers in view of concerns with combining QTc prolonging drugs. The SVT continued to be incessant but at lower rates of $120-130 \mathrm{bpm}$ as an outpatient and the patient was monitored closely for the first 2 months with a 30 day remote telemetry monitor(Biotel, Malvern PA) and an owlet sock (Owlet ${ }^{\mathrm{TM}}$, Lehi, UT). Serial outpatient echocardiograms showed that at the two month checkup the infant's ejection fraction had dropped from $60 \%$ at discharge at three weeks of age to $50 \%$. The infant did not return for follow up for the 2.5 month check due to inter current viral respiratory illnesses in the family and the home monitors showed that the average heart rates were increasing from the $120 \mathrm{~s}$ to the $140 \mathrm{~s}$. At three months he presented to the ER with the above picture. He was started on milrinone and furosemide and from an antiarrhythmic standpoint, was loaded with digoxin, another short load of amiodarone PO followed by maintenance amiodarone and his beta blockers increased. Flecainide was discontinued in view of the deterioration in cardiac function. On this regimen he had an unresponsive staring episode that lasted a few seconds. His heart rates were low in the 70s and his digoxin level was elevated at 4.1. A decision was made to take him to the Electrophysiology laboratory for a catheter ablation. $72 \mathrm{hrs}$ prior to the planned ablation the oral amiodarone, digoxin and beta blockers were stopped and he was placed an on esmalol infusion to keep his heart rates under $130 \mathrm{bpm}$. The esmalol was titrated upto 150 microgram $/ \mathrm{kg} / \mathrm{minute}$ and was stopped 30 mins prior to the ablation.

EP study:

The EP study was performed under general anesthesia on inotropic support. The infant was in incessant SVT in the EP lab. A 3 catheter study was performed with access through the right and left femoral veins. $3 \mathrm{D}$ electroanatomical mapping was performed with the EnSite Precision mapping system (Abbott Medical, Inc, St Paul, MN). A 4 Fr decapolar (Inquiry, Abott Medical, Inc) was placed in the CS and a 4 Fr quadripolar catheter (JSN TM, Abbott Medical, Inc) was placed in the right ventricular apex. Mapping was performed 
with a 5 Fr Marinr small curve catheter (Medtronic, Inc., Minneapolis, MN, USA). Atrial extrastimuli resulted in transient interruption followed by resumption after two to three sinus beats. His bundle-refractory PVCs during tachycardia delayed atrial activation. Entrainment during SVT was performed with pacing in the RV apex and the atrial activation sequence during pacing was identical to the tachycardia. It was clear early on in the case that the pathway location was close to the CS dipoles 7.8 within the CS. Detailed mapping of the right septum and postero-septal space was performed and the Mariner was inserted into the CS alongside the decapolar catheter. Earliest atrial activation during SVT was noted in a diverticulum off the os of the CS (Figure 1). An aortic root shot was performed through a 4 Fr pigtail catheter in the RFA to delineate coronary anatomy. The catheter tip was distinct from the main coronary arteries and branches. Current delivery with a low power 7 Watt lesion resulted in an immediate temperature increase to 50 degrees Celsius, impedance of 115 ohms and cessation of the SVT within 1.3 seconds (Figure 2). This was evidenced by a change in heart rate and CS activation pattern. The lesion was applied for a full 40 seconds. No insurance burns were applied. A full EP study was then performed in sinus rhythm and was normal. Repeated electrical stimulation was performed with atrial and ventricular single and doubles at coupling intervals down to $180 \mathrm{msec}$ on and off Isoproterenol and during the washout phase of the drug with no evidence of SVT. 60 minutes post ablation the catheter were removed.

Post ablation course: The patient was transferred back to the ICU with continuous telemetry monitoring. Milrinone was stopped 12 hours post ablation and an echocardiogram 48 hours later showed an improvement in $\mathrm{EF}$ to $45 \%$. The patient continued to be in sinus rhythm three days later and was discharged home on a home telemetry monitor. Six month later the patient is doing very well with completely recovered cardiac function off of medications.

\section{Discussion}

PJRT is an uncommon but important causes of refractory SVT in infants and children. ${ }^{5,6}$ These accessory pathway typically have only retrograde conduction properties and are slow and decremental. The pathway characteristics are similar to AV nodal tissue and therefore the circuit has two decrementally conducing limbs making for stable reentrant SVT at varying cycle lengths. ${ }^{2-4}$

Medical management can be difficult and poor rhythm and or rate control can lead to cardiac decompensation and tachycardia induced cardiomyopathy. These pathways are most commonly located in the right septal area, more commonly near the posterior septum but mid or anterior septal locations have also been described. The right posteroseptal pathways may be close to the ostium of the CS and may be within the proximal CS but locations have been described in almost any position along the AV groove. ${ }^{3,5,6}$ However epicardial connections distant from the mitral and tricuspid valve annulus within a diverticulum of the CS are unusual and to the best of our knowledge an ablation in this location has not been previously described in an infant.

We opted to perform an aortic root shot in our patient prior to RF application as ablation of accessory pathways from within the CS may lead to damage to nearby coronary arteries (CAs) and their branches. Specifically the posterolateral (inferolateral) ventricular branch of the right coronary artery and the left circumflex artery run inferiorly and in close proximity to the CS. Use of radiofrequency catheter ablation within the CS has been reported to cause ischemia and infarction due to stenosis or complete occlusion. Symptoms may be acute and catastrophic or present several weeks later. ${ }^{7}$

Other complications described with RF lesions within the CS include cardiac tamponade, AV block, and pericarditis and our infant went home on a 30 day event recorder to monitor for late onset heart block. ${ }^{8}$

In our patient we choose to deliver a low power RF burn at $7 \mathrm{~W}$, to avoid perforation of this tiny CS branch. Kusano et al have described low power $10 \mathrm{~W}$ lesions in the coronary sinus achieving temperatures of 60 degrees. ${ }^{8}$

\section{Conclusion}

Tachycardia induced cardiomyopathy can be reversed with RF ablation. Low power burns in the CS are effective in achieving adequate temperatures. Lesions in the coronary sinus are high risk and care must be 
taken to delineate coronary anatomy.

Funding: This research did not receive any specific grant from funding agencies in the public, commercial, or not-for-profit sectors.

\section{References:}

1. Josephson ME. Supraventricular tachycardias. In: Josephson ME editor. Josephson's Clinical cardiac electrophysiology: techniques and interpretations. 5th ed. Philadelphia: Wolters Kluwer; 2016; p. 171-280.

2. Coumel P, Cabrol C, Fabiato A. Tachycardie permanente par rythme réciproque. Preuves du diagnostic par stimulation auriculaire et ventriculaire. Arch Mal Coeur. 1967;60:1830-64.

3. Coumel P. Junctional reciprocating tachycardias. The permanent and paroxysmal forms of A-V nodal reciprocating tachycardias. J Electrocardiol. 1975;8:79-90.

4. Critelli G. Recognizing and managing permanent junctional re- ciprocating tachycardia in the catheter ablation era. J Cardiovasc Electrophysiol. 1997;8(2):226-36.

5. Vaksmann G, D'Hoinne C, Lucet V, Guillaumont S, Lupoglazoff JM, Chantepie A, et al. Permanent junctional reciprocating tachycardia in children: a multicenter study on clinical profile and outcome. Heart. 2006;92(1):101-4.

6. Kang KT, Potts JE, Radbill AE, La Page MJ, Papagiannis J, Garnreiter JM, Kubus P, Kantoch MJ, Von Bergen NH, Fournier A, Cote JM, Paul T, Anderson CC, Cannon BC, Miyake CY, Blaufox AD, Etheridge SP, Sanatani S. Permanent junctional reciprocating tachycardia in children: a multicenter experience. Heart Rhythm. 2014 Aug;11(8):1426-32. doi: 10.1016/j.hrthm.2014.04.033. Epub 2014 Apr 24.PubMed PMID: 24769425.

7. Schneider HE, Kriebel T, Gravenhorst VD, Paul T. Incidence of coronary artery injury immediately after catheter ablation for supraventricular tachycardias in infants and children. Heart Rhythm. 2009 Apr;6(4):461-7. doi:10.1016/j.hrthm.2009.01.029. Epub 2009 Feb 25. PubMed PMID: 19324303.

8. Jackman WM, Wang X, Friday KJ, Roman CA, Moulton KP, Beckman KJ, McClelland JH, Twidale N, Hazlitt HA, Prior MI. Catheter ablation of accessory atrioventricular pathways (Wolff-ParkinsonWhite syndrome) by radiofrequency current. N Engl J Med 1991;324: 1605-11

9. Kusano KF, Morita H, Fujimoto Y, Hirose E, Ohe T. Catheter ablation of an epicardial accessory pathway via the middle cardiac vein guided by monophasic action potential recordings. Europace. 2001 Apr;3 (2):164-7. PubMed PMID:11333058.

Figure 1: Location of catheter at successful ablation site in a diverticulum close to the os of the coronary sinus 


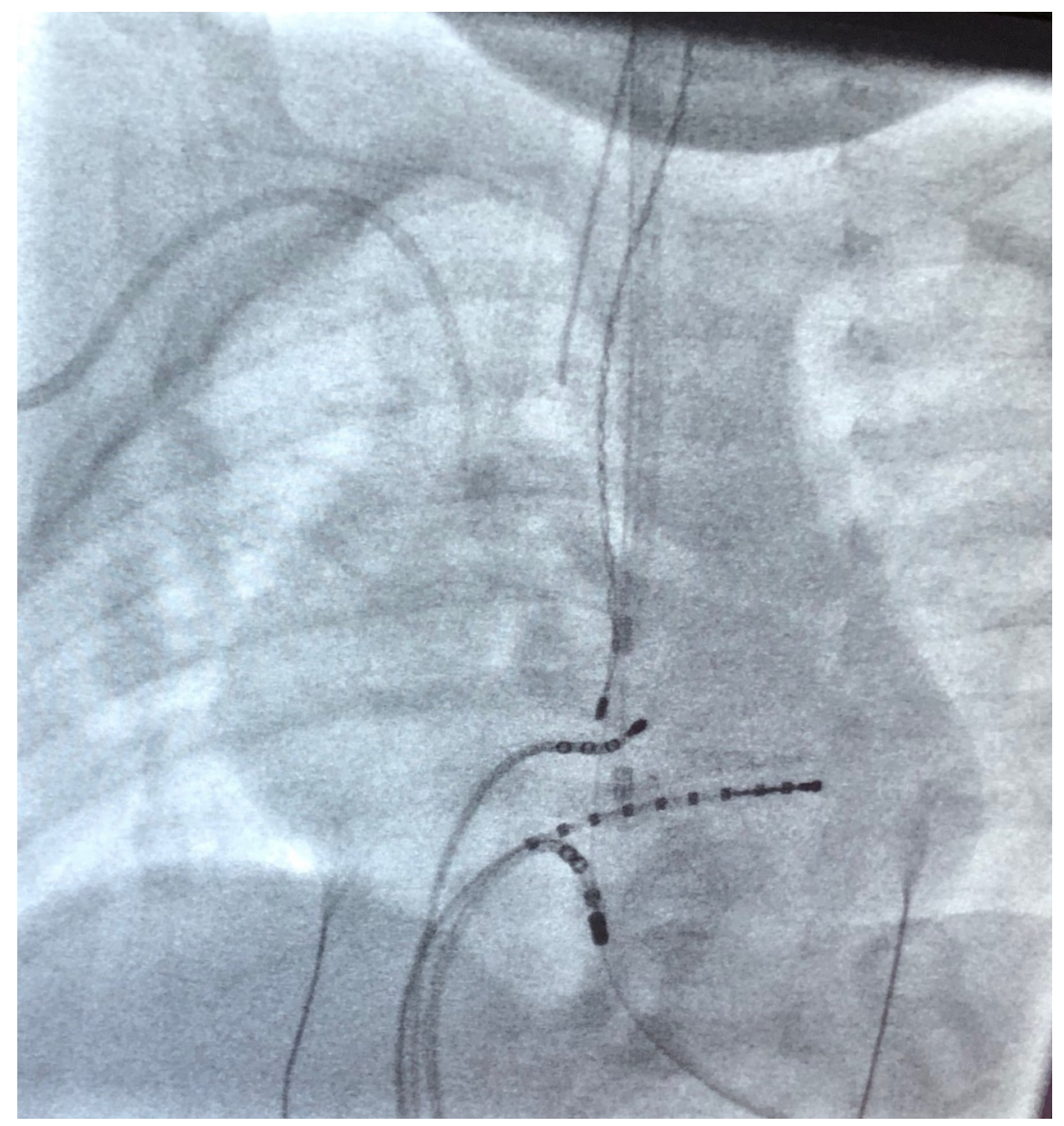

Figure 2: Termination of SVT with RF ablation. Note change in the cycle length, surface ECG and activation pattern in the CS dipoles post ablation.

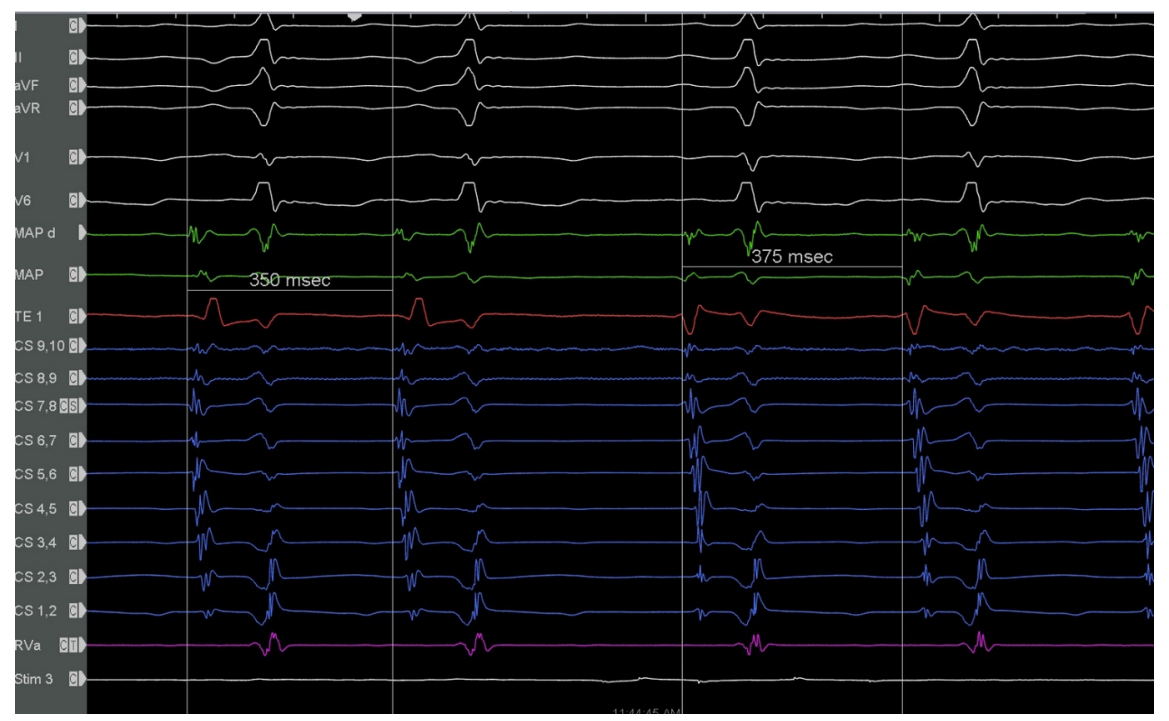

